\title{
K-shell ionization cross sections of atoms by muons and pions ${ }^{\star}$
}

\author{
Takeshi Mukoyama a and Károly Tőkési \\ Institute for Nuclear Research of the Hungarian Academy of Sciences (ATOMKI), Bem tér 18/c, 4026 Debrecen, Hungary
}

Received 14 November 2018 / Received in final form 27 March 2019

Published online 11 June 2019

(c) The Author(s) 2019. This article is published with open access at Springerlink.com

\begin{abstract}
The K-shell ionization cross sections of copper and silver atoms by muons and pions with negative and positive charge have been calculated with the classical trajectory Monte Carlo method and the planewave Born approximation with corrections for the Coulomb-deflection and binding-energy effects. Both results are in good agreement with each other. The obtained cross sections for muons on copper are also compared with the coupled-channels calculations. The dependence of the K-shell ionization cross sections on the sign of the projectile as well as on the projectile mass is discussed.
\end{abstract}

\section{Introduction}

The inner-shell ionization processes by charged-particle impact have been extensively studied both theoretically and experimentally as a basic process in atomic physics as well as applications to various fields, such as solid-state physics, radiation physics, astrophysics, plasma physics and chemistry [1]. It is well known that the experimental ionization cross sections in high-energy region can be described with the theoretical models based on the firstorder Born approximation (FBA), such as the plane-wave Born approximation (PWBA) [2] and the semi-classical approximation (SCA) [3]. In the FBA, the ionization cross section is proportional to $Z_{1}^{2}$, where $Z_{1}$ is the projectile charge. This fact means that the cross section does not depend on the charge of the projectile and the ionization cross sections for antiparticles are same as those for particles.

However, for low- and intermediate-energy projectiles the experimental values deviate from the FBA results and higher-order corrections should be introduced for accurate description of experimental observations. In the lowenergy region the projectile trajectory is deflected due to the Coulomb field of the target nucleus. In addition, the initial bound states of the target electrons are distorted due to the presence of the projectile and their binding energies are changed. On the other hand, at the intermediate and high velocities the polarization effect of the target electron orbital caused by the projectile becomes important. Owing to these effects the inner-shell ionization cross sections deviate from the simple $Z_{1}^{2}$ scaling law and change when the sign of the projectile changes. It is

* Contribution to the Topical Issue "Many Particle Spectroscopy of Atoms, Molecules, Clusters and Surfaces (2018)", edited by Károly Tőkési, Béla Paripás, Gábor Pszota, and Andrey V. Solov'yov.

a e-mail: hakutei@rhythm.ocn.ne.jp interesting to study the dependence of inner-shell ionization cross sections on the sign of the projectiles.

Earlier experimental investigations for comparison of inner-shell ionization cross sections between particles and antiparticles have been performed by the use of electrons and positrons [4-6]. The large difference in the K-shell ionization cross sections between electrons and positrons was observed at low energies and it was explained by the trajectory of the projectile in the Coulomb field [7].

Andersen et al. [8] measured K-shell ionization cross sections of helium by proton and antiprotons at the LowEnergy Antiproton Ring (LEAR) at CERN. They found large difference in the ratios of double to single K-shell ionization cross sections between protons and antiprotons, but their single K-shell ionization cross sections for positively and negatively charged particles are same within the experimental errors.

Since then the experimental and theoretical studies on particle and antiparticle collisions have been reported and the results for proton-antiproton pair as well as electronpositron pair have been discussed in many reviews [9-12]. It is well known that the difference in ionization cross sections depends on the energy and mass of the projectile as well as the target atomic number. However, almost all of the single ionization cross sections cited in the reviews are for the K-shell ionization cross sections of low- $Z$ targets, such as $\mathrm{H}$ and $\mathrm{He}$ atoms. There have been reported the data for rare gases, for example $\mathrm{Ne}, \mathrm{Ar}, \mathrm{Kr}$ and $\mathrm{Xe}$ [13], but they are only for total single ionization cross sections, i.e. the sum of ionization cross sections of all shells, and contributions from each shell are not separately measured.

It is interesting to study $\mathrm{K}$-shell ionization of high- $Z$ elements by different massive particle-antiparticle pairs, such as muon $\left(\mu^{ \pm}\right)$and pion $\left(\pi^{ \pm}\right)$. They are considered to be more favorable projectiles to study the dependence of the ionization cross sections on the projectile charge than the electron-positron and proton-antiproton pairs. In the case of electrons, the incident particle is identical to 
the target electron and the exchange effect is important in low-energy region. Practically the electron scattering is described as Møller scattering, while the positron scattering is known as the Bhabha scattering. On the other hand, the charge transfer channel is important for lowenergy proton-atom collisions and this process reduces the number of protons available for ionization by positively charged particles.

The number of theoretical calculations for inner-shell ionization cross sections by muons and pions is rather scarce. Martir et al. [14,15] calculated the K-shell ionization cross sections for positive and negative muons on copper in the energy range from 1 to $2 \mathrm{MeV}$ /amu by the use of the coupled-channels (CC) method. They showed that the Coulomb-deflection effect is quite large in comparison with the case for protons and antiprotons. Cohen [16] used the classical trajectory Monte Carlo (CTMC) method and calculated the ionization and capture cross sections for negative muons on hydrogen atom in the energy region between $3 \mathrm{eV}$ and $100 \mathrm{keV}$. The similar CTMC calculations were made by Hock et al. [17] for muons and pions on hydrogen.

Mukoyama et al. [18] performed the PWBA calculations for K-shell ionization cross sections of $\mathrm{Cu}, \mathrm{Ag}$ and $\mathrm{Au}$ by muons and pions. Recently Sakimoto [19] calculated ionization cross sections for negative muons on hydrogen by the use of quantum-mechanical wavepacket method and the semiclassical method at low energies. Except for the CC calculations [14,15] and our previous work [18] other theoretical calculations are for hydrogen atom. On the other hand, there have been reported no experimental results for inner-shell ionization cross sections by muons and pions.

The aim of the present work is to estimate the K-shell ionization cross sections for atoms heavier than He by muons and pions with negative and positive charge and to study the difference in the K-shell ionization cross sections for particle and antiparticle collisions. We use the CTMC and the modified PWBA methods. In the latter case, the binding-energy and polarization effect and the Coulomb-deflection effects are taken into account.

\section{Theoretical models}

In the present work, we calculate the K-shell ionization cross sections by positive and negative muons and pions with two different methods: (1) the CTMC method [20] and (2) the PWBA corrected for the binding-energy change of the target electron and the Coulomb-deflection effect of the projectile [21].

\subsection{Classical trajectory Monte Carlo method}

The CTMC method is a non-perturbative method based on the classical dynamics and all the interactions between the colliding particles are automatically taken into consideration. In order to apply the CTMC to the atoms other than hydrogen, we used the screened hydrogenic model and the screening constant was determined according to

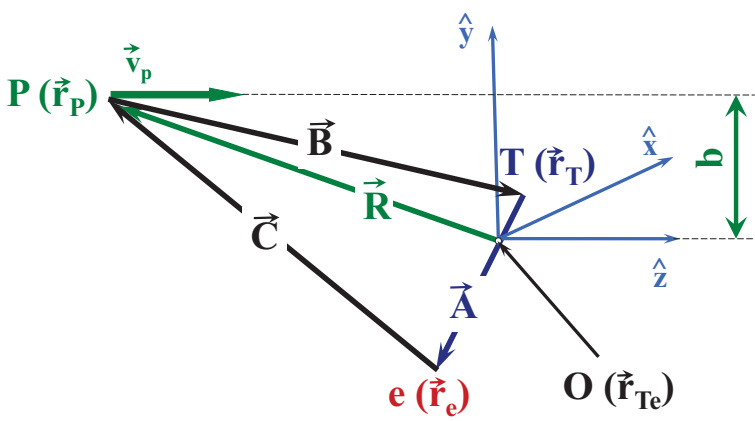

Fig. 1. Relative positions of the 3-body system used for the CTMC. The symbol $P$ is the projectile, $O$ the center of mass of the target, $T$ the target nucleus, $e$ the electron, and $b$ the impact parameter.

Slater's rule [22]. Then the system becomes as the threebody system, which consists of the projectile, the target nucleus and the target electron. They are assumed to interact with each other through the pure Coulomb field.

Figure 1 shows the relative position vectors of particles in the present 3-body system. The projectile $P$ is moving with the velocity $\boldsymbol{v}_{p}$. The symbol $O$ indicates the center of mass of the target system. The target nucleus is located at $T$ and the electron is at $e$. The impact parameter is shown as $b$.

The classical equations of motion by Newton for threebody system are solved numerically for large number of trajectories with given initial conditions. The initial conditions correspond to the impact parameter of the projectile, the position and velocity of the target electrons. For each trajectory, these parameters are selected randomly by the use of pseudo-random numbers.

With each initial condition thus determined, the equations of motion for the system are solved by numerical integration with respect to time using the standard Runge-Kutta method. In order to distinguish the various final states, the exit channels were tested at large distances from the collision center. For positively charged particles, the electron capture process was included in the final channel, but its contribution was small. For the ionization channel, the relative two-body energies were tested among the particles. We consider that the obtained channel is ionization if all the three relative two-body energies are greater than 0 .

The total ionization cross section is obtained from

$$
\sigma=\frac{2 \pi b_{\max } \sum_{j} b_{j}^{(i)}}{N},
$$

where $N$ is the total number of trajectories with impact parameters less than $b_{\max }$ and $b_{j}^{(i)}$ is the actual impact parameter of the $j$-th trajectory corresponding to the ionization or capture channel. The standard deviation of the ionization cross section is expressed as

$$
\Delta \sigma=\sigma\left(\frac{N-N^{(i)}}{N N^{(i)}}\right)^{1 / 2},
$$

where $N^{(i)}$ is the number of trajectories which satisfy the criterion for the ionization process. 


\subsection{Modified plane-wave born approximation}

As mentioned above, the inner-shell ionization cross section in the PWBA is independent of the sign of the projectile. We use the ECPSSR model developed by Brandt and Lapicki [21], which goes beyond the FBA by introducing corrections for various effects into the PWBA.

The ECPSSR starts from the nonrelativistic PWBA cross sections with screened hydrogenic wave functions and takes into account the energy-loss effect $(\mathrm{E})$, Coulomb-deflection effect $(\mathrm{C})$, polarization and bindingenergy effect in the perturbed stationary state (PSS) and the electronic relativistic effect $(\mathrm{R})$. The present ECPSSR model is in principle same as the method used by Brandt and Basbas $[23,24]$ for protons and antiprotons.

In the ECPSSR, the K-shell ionization cross section is expressed as [21]

$$
\sigma_{K}=C_{K}\left(\frac{\pi d q_{0} \zeta}{z_{K}\left(1+z_{K}\right)}\right) \sigma_{K}^{\mathrm{PWBA}}\left(\zeta \theta, m^{R} \eta\right)
$$

where $C(x)$ is the multiplicative correction factor for the Coulomb-deflection effect, $d$ is one-half of the distance of the closest approach in head-on collision, $\hbar q_{0}$ is the minimum momentum transfer in ionization and $z_{K}$ is the parameter for the energy-loss correction.

The PWBA ionization cross section with approximate limits of momentum transfer for the scaled K-shell binding energy $\theta$ and the scaled projectile velocity $\eta$ is given by [2]

$$
\sigma_{K}^{\mathrm{PWBA}}(\theta, \eta)=\frac{8 \pi Z_{1}^{2}}{Z_{2 K}^{4} \eta} f(\theta, \eta) a_{0}^{2},
$$

where $Z_{2 K}$ is the effective nuclear charge of the target K-shell electron, $f(\theta, \eta)$ is the universal function with parameters $\theta$ and $\eta$ and $a_{0}$ is the Bohr radius of hydrogen.

In equation (3), $\zeta$ is the binding-energy and polarization correction factor and $m^{R}$ is the correction factor for the electronic relativistic effect of the target electron. For antiparticles we calculated the Coulomb-deflection factor with the procedure proposed by Brandt and Basbas $[23,24]$.

\section{Results and discussion}

In the case of the CTMC, a very large number of the classical trajectories were computed because the K-shell ionization cross sections decrease drastically with decreasing incident energy of the projectile. We followed 5-million histories for each impact energy.

The ECPSSR calculations were performed with the modified version of the DEKY code [25,26]. However, it should be noted that the cross section $\sigma_{K}^{\mathrm{PWBA}}(\theta, \eta)$ is calculated with approximate limits of momentum transfer and the correction for the energy-loss effect is made through the parameter $z_{K}$. We have shown [27] that when the relativistic correction is made through $m^{R} \eta$ in $f(\theta, \eta)$ with the exact limits of momentum transfer, called the CPSSR model, the electronic relativistic effect

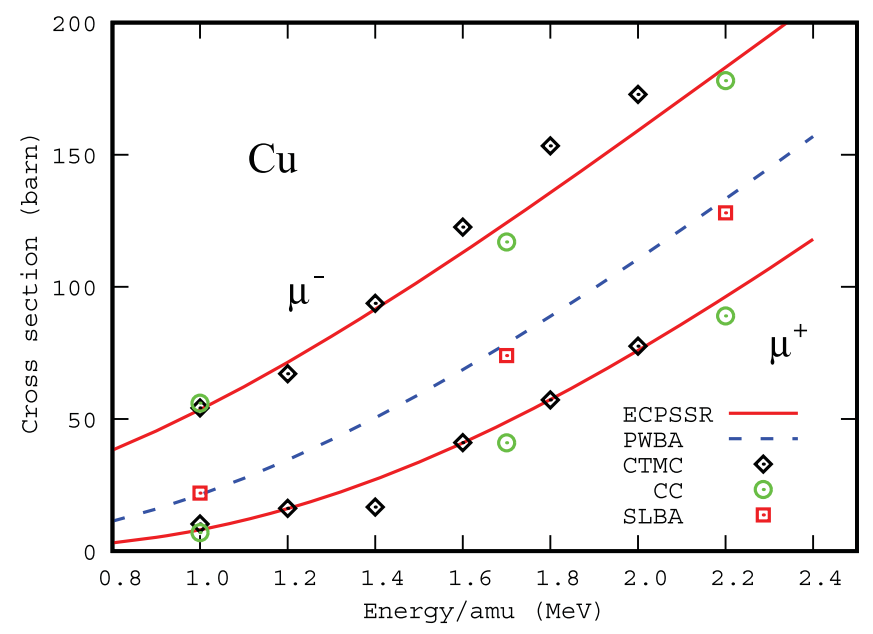

Fig. 2. K-shell ionization cross sections of $\mathrm{Cu}$ by positive and negative muon impact. The solid lines are the ECPSSR, the dashed lines the PWBA, the diamonds the CTMC, the circles the CC by Martir et al. [14], and the squares the SLBA by Reading et al. [15].

is overestimated at low energies and gives larger values for $\sigma_{K}^{\mathrm{PWBA}}(\theta, \eta)$.

In our previous work [18], where the CPSSR was used, the calculated cross sections are different from the ECPSSR ones at low energies. The validity for applications of the ECPSSR method to antiparticles has been tested by Lapicki [28].

We consider asymmetric collisions where the target atomic number is larger than that of the projectile and the models based on the perturbation theory are valid. In order to demonstrate the difference in ionization cross sections between particles and antiparticles, we choose the energy region where the projectile velocity is much smaller than the velocity of the target electron.

In Figure 2, K-shell ionization cross sections of $\mathrm{Cu}$ by negative and positive muons in the CTMC and the ECPSSR are shown as a function of projectile energy and compared with the results in the CC calculations [14,15]. For comparison, the PWBA cross sections, $\sigma_{K}^{\mathrm{PWBA}}(\theta, \eta)$, and the FBA calculations with straight-line trajectories (SLBA) by Reading et al. [15] are also plotted in the figure.

It is clear that the cross sections for positive muons are below than the PWBA ones, while those for negative muons are above. This is due to the Coulomb-deflection effect of the projectile and the binding-energy effect of the target electron. The present results in the CTMC and the ECPSSR approaches are in good agreement with each other and agree well also with the CC calculations [14,15]. On the other hand, the present PWBA calculations are in agreement with the SLBA of Reading et al. [15].

In Figure 3, we present the ratio of the K-shell ionization cross sections of $\mathrm{Cu}$ to the PWBA cross section $\sigma_{K}^{\mathrm{PWBA}}(\theta, \eta)$. The broken curves indicate the cross section without the Coulomb-deflection effect and can be called as the EPSSR. These results are in good agreement with the CC method with straight-line trajectory $[14,15]$. The deviation of these curves from unity is explained as the 


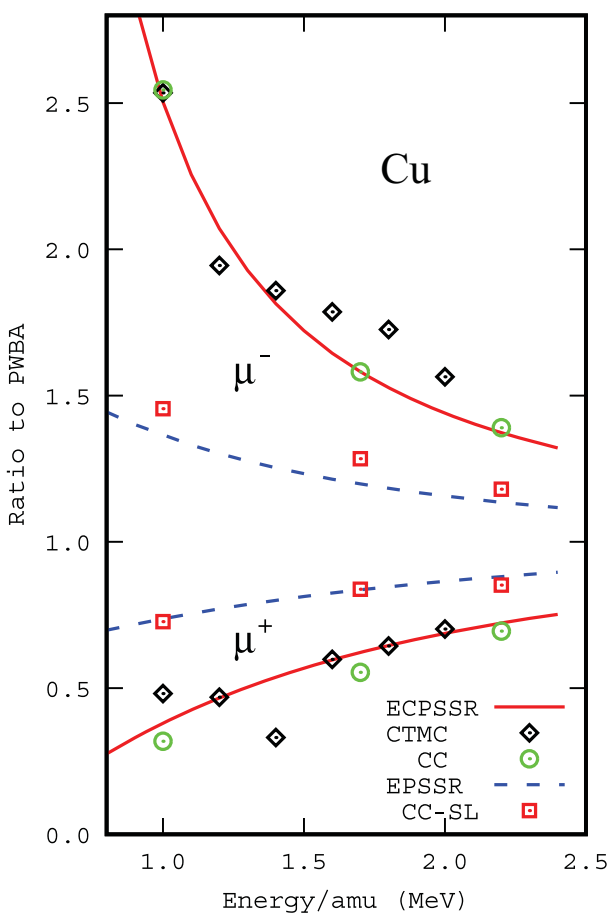

Fig. 3. Ratio of the calculated cross sections to the PWBA for positive and negative muons on $\mathrm{Cu}$. The solid lines are the ECPSSR, the dashed lines the EPSSR, the diamonds the CTMC, the circles the CC by Martir et al. [14], and the squares the CC with straight-line trajectory [14].

binding-energy effect of the target electron due to presence of the charged projectile. For negative muons, the relative cross sections are higher than unity and this fact corresponds to the decrease in the binding energy of the target electron. This "anti-binding" effect was first demonstrated by Reading et al. [15] for muons.

On the other hand, the difference between the ECPSSR and the EPSSR can be ascribed to the effect of the Coulomb interaction between the projectile and the target nucleus. For positive muons, the Coulomb interaction is repulsive and the ionization cross section is small, while it is attractive in the case of negative muons and increases the cross section. It can be seen from the figure that the Coulomb-deflection effect is larger than the bindingenergy effect.

When the target atomic number increases, the ionization cross section decreases for the projectile with the same velocity. In Figure 4, we plot the K-shell ionization cross sections of $\mathrm{Ag}$ by positive and negative muons. The calculations were made only with the ECPSSR, the EPSSR and the PWBA. The CTMC calculations were not performed because the ionization cross section in the present energy region is too small and we need huge number of trajectories to obtain reliable values. The results indicate that the binding-energy effect, EPSSR/PWBA, is almost same as the values for $\mathrm{Cu}$, but the Coulombdeflection effect is larger.

The K-shell ionization cross sections of $\mathrm{Cu}$ for positive and negative pion impact are shown in Figure 5. The CTMC calculations agree well with the ECPSSR. For

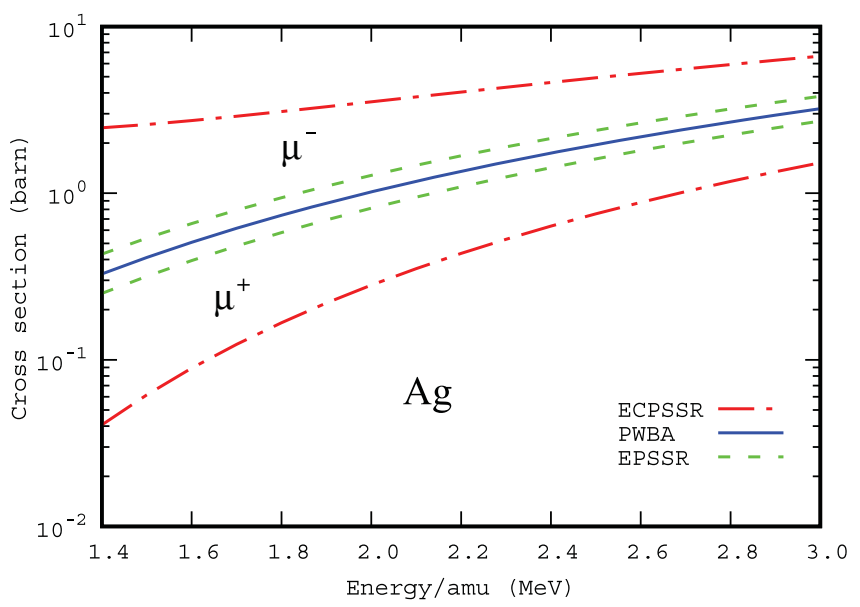

Fig. 4. K-shell ionization cross sections of $\mathrm{Ag}$ by positive and negative muon impact. The solid line is the PWBA, the dotdashed lines are the ECPSSR, and the dashed lines the EPSSR.

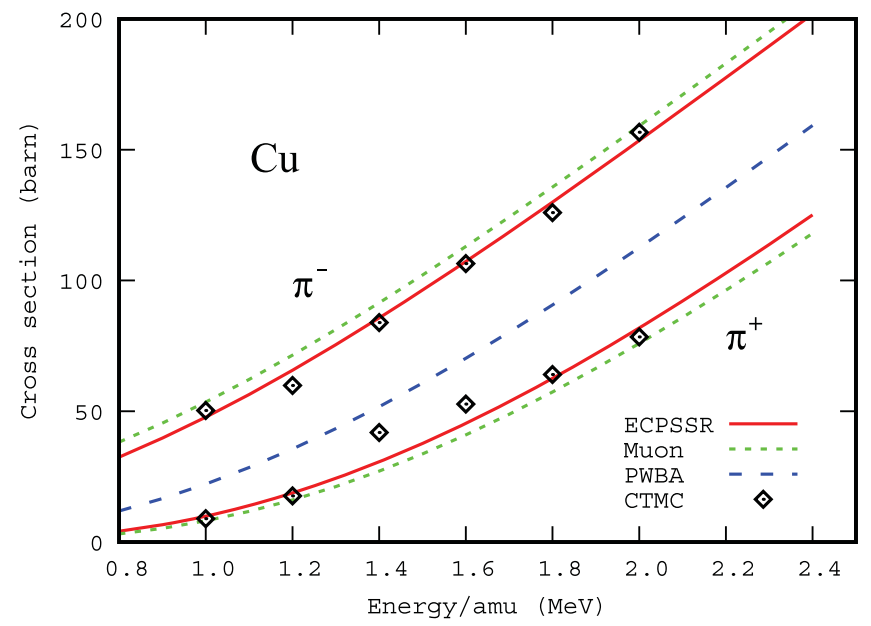

Fig. 5. K-shell ionization cross sections of $\mathrm{Cu}$ by positive and negative pion impact The solid lines are the ECPSSR, the dotted lines the ECPSSR for muons, the dashed line is the PWBA, and the diamonds are the CTMC.

comparison, the present results for muons in the ECPSSR are also plotted in the figure. When the projectile energy is expressed as energy per amu, the PWBA cross sections are almost same those for muons. Owing to small difference in the parameters for the energy-loss effect, the cross sections for positive pions are slightly larger than those for muons, $4 \%$ at $1 \mathrm{Mev} / \mathrm{amu}$ and $2 \%$ at $2 \mathrm{MeV} / \mathrm{amu}$.

For same projectile velocity, the binding-energy correction for the PWBA is same and the ratio of the EPSSR to the PWBA is equal for muons and pions. On the other hand, the correction factor for the Coulomb-deflection effect is different between muons and pions. The difference in the ECPSSR cross sections for muons and pions in Figure 5 is ascribed to the effect of the Coulomb trajectory of the projectile. Because of larger mass, the ionization cross sections by positive pions are larger than those by positive muons, while for negative particles the ECPSSR values for pions are smaller than those for muons. 


\section{Conclusion}

The K-shell ionization cross sections by positively- and negatively-charged muons and pions have been calculated at low energies with the CTMC and the ECPSSR. The obtained results are in good agreement with each other and also with the $\mathrm{CC}$ calculations for muons on $\mathrm{Cu}$.

The ECPSSR cross sections by negative projectiles are larger than the PWBA and the values for positive projectiles are in the opposite direction. The reasons can be ascribed to the binding-energy effect of the target electron and the Coulomb-deflection effect of the projectile. In the energy region considered here the latter effect is dominant.

At the same projectile velocity the cross section for pions is almost same as that for muons, but the deviation from the PWBA is slightly larger for muons. This is explained as the difference in the Coulomb-deflection effect.

At present the quality of beams for muons and pions is not enough to measure the K-shell ionization cross sections for elements calculated here by these projectiles. However, it is hoped that the present results provide useful benchmark values for more elaborate theoretical models and stimulate experimental studies for K-shell ionization cross sections by particles and antiparticles in future.

Open access funding provided by MTA Institute for Nuclear Research (MTA ATOMKI). This work was supported by the National Research, Development and Innovation Office (NKFIH) under Grant KH 126886 and the European Cost Actions CA15107 (MultiComp) and MP1306 (MPNS).

\section{Author contribution statement}

T.M. made the ECPSSR calculations and wrote most of the manuscript. K.T. performed the CTMC calculations and prepared Figure 1.

Open Access This is an open access article distributed under the terms of the Creative Commons Attribution License (http://creativecommons.org/licenses/by/4.0/), which permits unrestricted use, distribution, and reproduction in any medium, provided the original work is properly cited.

\section{References}

1. E.W. McDaniel, J.B.A. Mitchell, M.E. Rudd, Atomic Collisions: Heavy Particle Projectiles (Wiley, 1993)
2. F. Merzbacher and H. Lewis, in Handbuch der Physik, edited by D. Flügge (Springer, 1958), Vol. 34, pp. 166-192

3. J. Bang, J.M. Hansteen, Kgl. Dansk. Mat. Fys. Medd. 31, 13 (1959)

4. S. Ito, S. Shimizu, T. Kawaratani, K. Kubota, Phys. Rev. A 22, 407 (1980)

5. P.J. Schmidt, J.L. Campbell, Phys. Lett. A 112, 316 (1985)

6. F. Ebel, W. Faust, C. Hahn, M. Rückert, H. Schneider, A. Singe, I. Toburen, Phys. Lett. A 140, 114 (1989)

7. G. Hock, in Abstracts of Contributed Papers of the XIth International Conference on the Physics of Electronic and Atomic Collisions, Kyoto, August 29-September 4, 1979, edited by K. Takayanagi, N. Oda (North Holland, 1979), p. 974

8. L.H. Andersen, P. Hvelplund, H. Knudsen, S.P. Møller, A.H. Sørensen, K. Elsener, K.G. Rensfelt, E. Uggerhøj, Phys. Rev. A 36, 3612 (1987)

9. H. Knudsen, J.F. Reading, Phys. Rep. 212, 107 (1992)

10. G. Laricchia, M. Charton, Philos. Trans. R. Soc. London A 357, 1259 (1997)

11. H. Knudsen, Hyperfine Interact. 109, 133 (1997)

12. T. Kirchner, H. Knudsen, J. Phys. B: At., Mol. Opt. Phys. 44, 122001 (2011)

13. K. Paludan, G. Laricchia, P. Ashley, V. Kara, J. Moxom, H. Blume, H. Knudsen, U. Mikkelsen, S.P. Møller, E. Uggehøj, E. Morenzoni, J. Phys. B: At., Mol. Opt. Phys. 30, L581 (1997)

14. M.H. Martir, A.L. Ford, J.F. Reading, R.L. Becker, J. Phys. B: At., Mol. Opt. Phys. 15, 1729 (1982)

15. J.F. Reading, A.L. Ford, M. Martir, R.L. Becker, Nucl. Instrum. Methods 192, 1 (1982)

16. J.S. Cohen, Phys. Rev. A 27, 167 (1983)

17. G. Hock, K. Tőkési, H. Nakamatsu, T. Mukoyama, ATOMKI Ann. Rep. 1991, 69 (1991)

18. T. Mukoyama, H. Kaji, K. Yoshihara, Bull. Inst. Chem. Res. Kyoto Univ. 68, 177 (1990)

19. K. Sakimoto, J. Phys. B: At., Mol. Opt. Phys. 35, 997 (2002)

20. K. Tőkési, G. Hock, Nucl. Instrum. Methods Phys. Res. B 124, 398 (1997)

21. W. Brandt and G. Lapicki, Phys. Rev. A 23, 1717 (1981)

22. J.C. Slater, Phys. Rev. 36, 57 (1930)

23. W. Brandt, G. Basbas, Phys. Rev. A 27, 578 (1983)

24. W. Brandt, G. Basbas, Phys. Rev. A 29, 3142 (1983)

25. T. Mukoyama, L. Sarkadi, Bull. Inst. Chem. Res. Kyoto Univ. 58, 60 (1980)

26. T. Mukoyama, L. Sarkadi, Bull. Inst. Chem. Res. Kyoto Univ. 60, 67 (1982)

27. T. Mukoyama, Int. J. PIXE 8, 225 (1998)

28. G. Lapicki, Nucl. Instrum. Methods Phys. Res. B 241, 34 (2005) 